\title{
The owl and the pussycat: Gaze cues and visuospatial orienting
}

\author{
SUSANNE QUADFLIEG and MALIA F. MASON, \\ Dartmouth College, Hanover, New Hampshire \\ and \\ C. NEIL MACRAE \\ University of Aberdeen, Aberdeen, Scotland
}

\begin{abstract}
Recent research has shown that nonpredictive gaze cues trigger reflexive shifts in attention toward the looked-at location. But just how generalizable is this spatial cuing effect? In particular, are people especially tuned to gaze cues provided by conspecifics, or can comparable shifts in visual attention be triggered by other cue providers and directional cues? To investigate these issues, we used a standard cuing paradigm to compare the attentional orienting produced by different cue providers (i.e., animate vs. inanimate) and directional cues (i.e., eyes vs. arrows). The results of three experiments revealed that attentional orienting was insensitive to both the identity of the cue provider and the nature of the triggering cue. However, compared with arrows, gaze cues prompted a general enhancement in the efficiency of processing operations. We consider the implications of these findings for accounts of reflexive visual orienting.
\end{abstract}

Understanding the language of eyes is an exquisitely adapted human skill. From simple gaze cues, it is possible to compute not only the direction of social attention, but also the behavioral intentions, desires, and preferences of others (Baron-Cohen, 1995; Emery, 2000; Kleinke, 1986; Macrae, Hood, Milne, Rowe, \& Mason, 2002). As people naturally look at objects in the environment that are of interest or importance to them, gaze direction is a communicative channel of considerable signal value. It comes as no surprise, therefore, to learn that a specialized processing system is believed to deal with the problem of gaze detection (Allison, Puce, \& McCarthy, 2000; Haxby, Hoffman, \& Gobbini, 2000). Both electrophysiological and neuroimaging work have suggested that key aspects of this system are localized in regions of the superior temporal sulcus (Hoffman \& Haxby, 2000; Perrett, Hietanen, Oram, \& Benson, 1992; Perrett et al., 1985; Puce, Allison, Bentin, Gore, \& McCarthy, 1998; Schuller \& Rossion, 2001; Wicker, Michel, Henaff, \& Decety, 1998). But once gaze cues have been detected, are there any discernible behavioral consequences for perceivers? Put simply, if one observes a person looking toward a specific spatial location, does this impact on one's own behavior in any systematic, meaningful way?

The authors thank David Balota, Gillian Rhodes, and two anonymous reviewers for their comments on this work. Correspondence concerning this article should be addressed to N. Macrae, School of Psychology, University of Aberdeen, King's College, Aberdeen, Scotland (e-mail: c.n.macrae@abdn.as.uk).
The message that emerges from research on this topic is quite straightforward. Through the phenomenon of reflexive visual orienting (Posner, 1980), gaze cues exert a direct influence on behavior. When visual attention is directed toward a specific spatial location, stimuli presented to that location are detected more rapidly and accurately than when they are presented elsewhere in the environment. It is in this respect that gaze cues are important, since they have been shown to trigger reflexive shifts in visual attention to cued (i.e., looked at) locations, an effect that emerges for adults, infants, and rhesus macaques (see Deaner \& Platt, 2003; Driver et al., 1999; Friesen \& Kingstone, 1998, 2003; Hietanen, 1999; Hood, Willen, \& Driver, 1998; Kingstone, Friesen, \& Gazzaniga, 2000; Langton \& Bruce, 1999). That the eyes trigger such exogenous shifts in attention has been attributed to the biological relevance of gaze cues in social communication (Baron-Cohen, 1995; Emery, 2000; Friesen \& Kingstone, 1998). Since averted gaze can signal the appearance of a predator or dinner in the immediate environment (Langton, Watt, \& Bruce, 2000), the capacity to reflexively follow the attention of others is clearly advantageous.

Notwithstanding the demonstration that gaze cues reliably trigger attentional orienting (Friesen \& Kingstone, 1998; Langton et al., 2000), questions remain regarding the status of this cuing effect. For example, does the identity of the cue provider impact the emergence of attentional orienting? Can similar effects be elicited by biologically irrelevant directional cues, such as arrows (see Ristic, Friesen, \& Kingstone, 2002; Tipples, 2002)? To date, reflexive visual orienting in response to gaze cues has been demon- 
strated for both schematic (e.g., Friesen \& Kingstone, 1998, 2003) and real (Langton \& Bruce, 1999; Schuller \& Rossion, 2001) faces, but it is nonetheless unclear whether stimuli (i.e., cue providers) other than human faces prompt the emergence of this effect (Deaner \& Platt, 2003). For example, would cue providers from other species (e.g., dogs, cats, rabbits) also trigger attentional orienting? Interestingly, inspection of the relevant literature gives rise to competing predictions regarding this matter.

Given the biological importance of conspecifics (i.e., people are more important communicative channels than rabbits), it is possible that reflexive attentional orienting may be most pronounced when triggered by other people. It has been suggested that because infants arrive in the world prearmed with information about the structure of human faces (e.g., CONSPEC and CONLERN: see Morton \& Johnson, 1991) they demonstrate a preference for facelike stimuli (Goren, Sarty, \& Wu, 1975; Johnson, Dziurawiec, Ellis, \& Morton, 1991). As Morton and Johnson (1991) have argued, "CONLERN builds a representation that enables the infant to discriminate the human face from other stimuli and especially from the faces of other species" (p. 178). Thus, if humans are hardwired to be responsive to human faces, reflexive attentional orienting may be strongest when gaze cues are provided by conspecifics.

A competing possibility, however, is that gaze cues may operate independently of the identity of the cue provider. Perrett et al. (1985), for example, investigated the responsiveness of cells in the superior temporal sulcus of macaque monkeys to gaze cues provided by humans, other monkeys, and dolls. Importantly, cells were responsive to specific gaze configurations regardless of the identity of the cue provider. That is, cells that responded to an averted gaze in other monkeys were simi- larly responsive to a similar gaze direction in dolls or humans. It is therefore possible that reflexive attentional orienting may be an adaptive mechanism driven by gaze cues alone, with the cue provider exerting little impact on the emergence or magnitude of the effect (see Deaner $\&$ Platt, 2003). This viewpoint is supported by the observation that chimpanzees and dogs respond to gaze cues provided by human experimenters (Call, Hare, \& Tomasello, 1998; Miklosi, Polgardi, Topal, \& Csanyi, 1998; Povinelli \& Eddy, 1996, 1997; Tomasello, Call, \& Hare, 1998). In addition, orienting effects have been observed when only eyes or eyelike stimuli have been presented to participants (von Grünau \& Anston, 1995). Thus, on the basis of findings such as these, one may not expect attentional orienting in humans to be restricted to situations in which the cue providers are conspecifics.

To investigate the aforementioned possibilities, we used a standard cuing paradigm (e.g., Friesen \& Kingstone, 1998 ) to compare the reflexive orienting produced by various animate and inanimate cue providers (Experiments 1 and 2). Participants were required to respond to peripheral target items that were preceded by nonpredictive gaze cues. The identity of the cue provider was manipulated, such that gaze cues on specific trials were provided by another person (i.e., a conspecific) or a chimpanzee, tiger, owl, apple, or glove. Extending the current inquiry, we also considered the effects of different directional cues (i.e., eyes vs. arrows) on the emergence of attentional orienting (Experiment 3).

\section{EXPERIMENT 1}

\section{Method}

Participants and Design. Twenty-four undergraduates (21 women and 3 men) from Dartmouth College completed the exper-

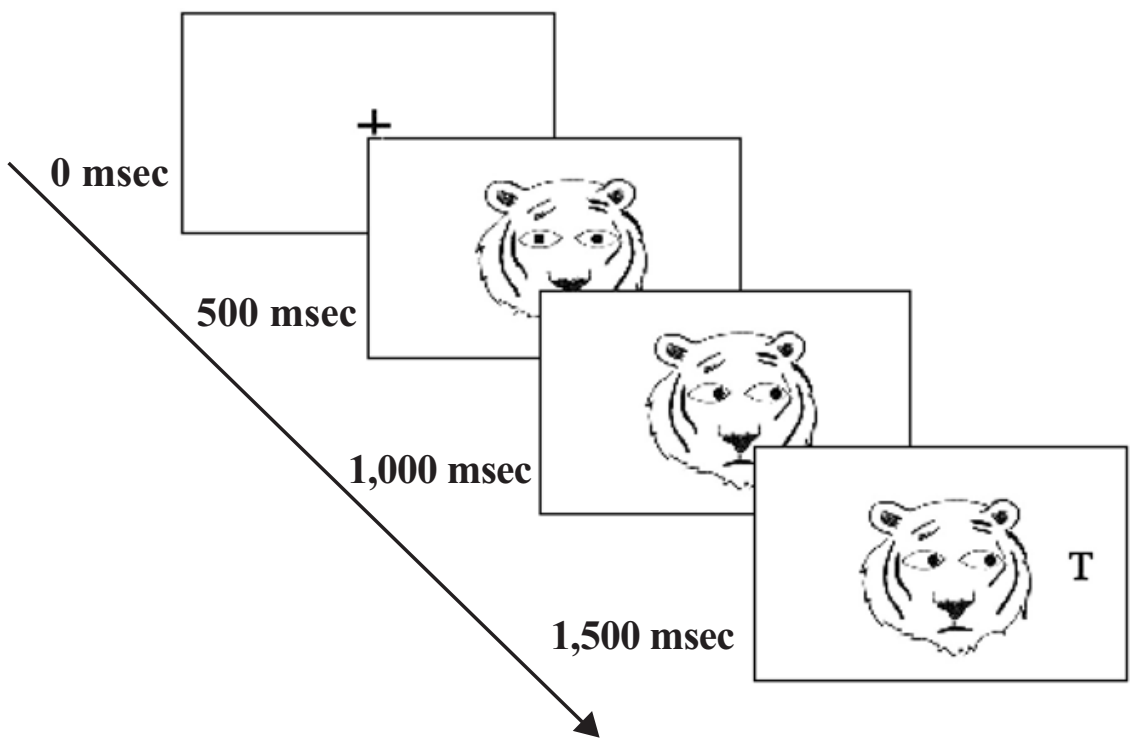

Figure 1. Stimulus presentation sequence illustrating a congruent trial (i.e., target appears at the cued location). 
iment for course credit. All participants had normal or corrected-tonormal vision. The experiment had a 2 (cue type: valid or invalid) $\times$ 4 (cue provider: person, chimpanzee, owl, or tiger) repeated measures design.

Stimulus, Materials, and Procedure. The experimental stimuli were presented and responses recorded using PsyScope software. The experiment was conducted on an iMac Macintosh computer with a 12 -in. monitor. Participants were seated approximately $57 \mathrm{~cm}$ from the monitor, and a chinrest was used to minimize head movements. The stimuli comprised the schematic faces of a person, chimpanzee, owl, and tiger (see Figure 1 for an example of one of the stimuli). Each face subtended a visual angle of $6^{\circ}$ horizontally and $6^{\circ}$ vertically. The faces were scanned in Adobe Photoshop and displayed as 8-bit grayscale images. The same template face was used for the three gaze configurations (i.e., direct, averted-left, averted-right). The images with eyes averted to the left or right differed only by a mirror reflection of the eye region. Adobe Photoshop was used to create the different versions of the three gaze configurations. The target letters were an uppercase $L$ or $T$ (visual angle: $0.75^{\circ} / 0.75^{\circ}$ ) and centered $4.8^{\circ}$ from the fixation cross on one side of the screen or the other.

A trial consisted of the following sequence of events (see Figure 1). A fixation cross appeared in the center of the screen for $500 \mathrm{msec}$. This image was then erased and replaced by a face (person, chimpanzee, owl, or tiger) displaying direct gaze for $500 \mathrm{msec}$, followed by the same face displaying gaze averted to the left or right, also for $500 \mathrm{msec}$. A target letter $(L$ or $T)$ then appeared on either the left or the right side of the screen and remained until participants made a response (i.e., cue/letter stimulus onset asynchrony $[\mathrm{SOA}]=500 \mathrm{msec})$. The letter was aligned either congruent to or opposite the gaze cue. In keeping with Driver et al.'s (1999) design, participants were asked to identify the target letter as quickly and accurately as possible by pressing the $H$ key on the computer keyboard with the index finger of their preferred hand if the letter was a $T$ or by pressing the spacebar with the thumb of the same hand if the letter was an $L$. The keys and letter mappings were counterbalanced across participants. It was stressed to participants that the central stimulus (i.e., the face) was irrelevant to the task and was not predictive of the location of the letter on each trial. Throughout the experiment, participants were instructed to fixate on the cross that appeared in the center of the screen. Participants completed 320 trials, with order of presentation of the cue provider, gaze direction, and target location randomized across the experiment. In total, participants completed 160 valid trials (40 for each cue provider) and 160 invalid trials (40 for each cue provider). On completion of the task, participants were debriefed, thanked, and dismissed.

\section{Results and Discussion}

Errors occurred on $7.4 \%$ of the trials and were excluded from the statistical analysis. Median response times were calculated for each of the experimental conditions and submitted to a 2 (cue type: valid or invalid) $\times$ 4 (cue provider: person, chimpanzee, owl, or tiger) repeated measures analysis of variance (ANOVA). The only effect to emerge in this analysis was a main effect of cue type $[F(1,23)=24.27, p<.001]$, indicating that participants returned faster responses on cued than on noncued trials. Neither the main effect of cue provider $[F(3,23)=1.78$, n.s. $]$ nor the cue type $\times$ cue provider interaction $[F(3,23)<1$, n.s. $]$ was significant. Thus, the pattern of responses clearly reveals that the identity of the cue provider did not moderate gaze-induced attentional orienting. Whether the triggering stimulus was a person, chimpanzee, owl, or tiger, the magnitude of attentional orienting was equivalent (see Figure 2). This finding suggests that gaze cuing is independent of the identity of the cue provider. Thus, even inanimate objects may trigger visuospatial orienting, if they possess eyelike stimuli and display the appropriate patterns of gaze motion. We investigated this possibility in our second experiment.

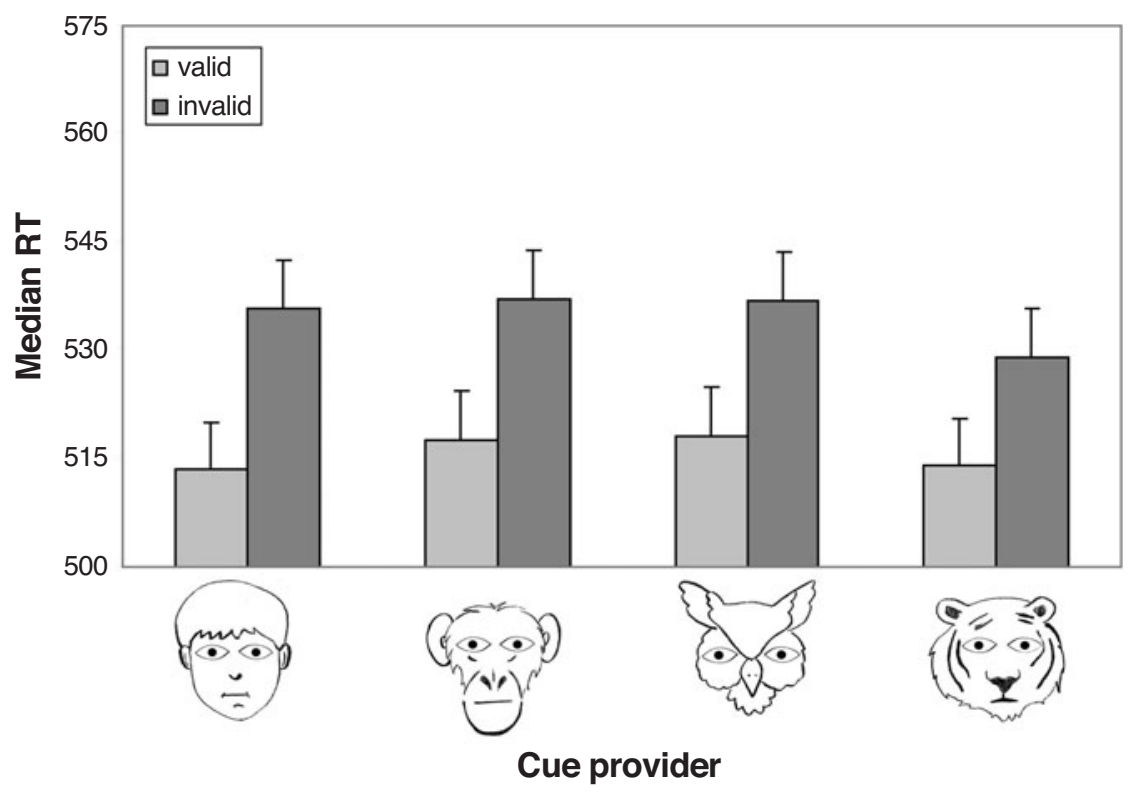

Figure 2. Median response time (in milliseconds) as a function of cue provider and trial type in Experiment 1. 


\section{EXPERIMENT 2}

\section{Method}

Participants and Design. Twenty-seven undergraduates (14 women and 13 men) from Dartmouth College completed the experiment for course credit. All participants had normal or corrected-tonormal vision. The experiment had a 2 (cue type: valid or invalid) $\times$ 4 (cue provider: person, chimpanzee, apple, or glove) repeated measures design.

Stimulus, Materials, and Procedure. In this experiment, gaze cues were provided by both animate (i.e., person, chimpanzee) and inanimate (i.e., apple, glove) cue providers. Participants completed 320 trials, with order of presentation of the cue provider, gaze direction, and target location randomized across the experiment. In total, participants completed 320 trials, 160 valid trials ( 80 animate and 80 inanimate cue providers) and 160 invalid trials ( 80 animate and 80 inanimate cue providers). On completion of the task, participants were debriefed, thanked, and dismissed.

\section{Results and Discussion}

Errors occurred on $6.8 \%$ of the trials and were excluded from the statistical analysis. The data from one male participant were also omitted from the analysis because of an excessive error rate. Median response times were calculated for each of the experimental conditions and submitted to a 2 (cue type: valid or invalid) $\times 4$ (cue provider: person, chimpanzee, apple, or glove) repeated measures ANOVA. The only effect to emerge in this analysis was a main effect of cue type $[F(1,25)=37.40$, $p<.001]$, indicating that participants returned faster responses on cued than on noncued trials. Neither the main effect of cue provider $[F(3,23)<1$, n.s. $]$ nor the cue type $\times$ cue provider interaction $[F(3,23)<1$, n.s.] was significant. Thus, the pattern of responses reveals that the animacy of the cue provider did not moderate gazeinduced attentional orienting. Whether the source of the triggering stimulus was a person, chimpanzee, apple, or glove, the magnitude of attentional orienting was equivalent (see Figure 3).

But what about the nature of the directional cues that prompt visuospatial orienting? Do only biologically relevant cues (e.g., eyes) trigger such effects? Recent research has suggested that eyes are not the only stimuli that produce shifts in visual attention, because symbolic directional cues such as arrows also trigger visuospatial orienting. Tipples (2002), for example, has shown that arrows can automatically trigger the orientation of attention to cued environmental locations (see also Ristic et al., 2002). Given this demonstration, researchers have questioned the assumption that gaze cues have a special signaling status in the human attentional system (i.e., that they exert qualitatively unique effects on attentional orienting). Even if they do not exert unique effects on spatial cuing, however, gaze cues may nevertheless promote quantitative differences in the efficiency of attentional processing. For example, whereas both biological (e.g., eyes) and nonbiological (e.g., arrows) cues may trigger attentional orienting, these effects may be initiated more efficiently (i.e., more rapidly) by the former than by the latter stimuli (Ristic et al., 2002). If operating, such an effect would reflect the evolutionary significance of gaze-related information compared with other directional cues in social-cognitive functioning (Kingstone, Smilek, Ristic, Friesen, \& Eastwood, 2003). We explored this issue in our third experiment.

\section{EXPERIMENT 3}

\section{Method}

Participants and Design. Ten undergraduates (4 women and 6 men) from Dartmouth College completed the experiment for course credit. All participants had normal or corrected-to-normal vision.

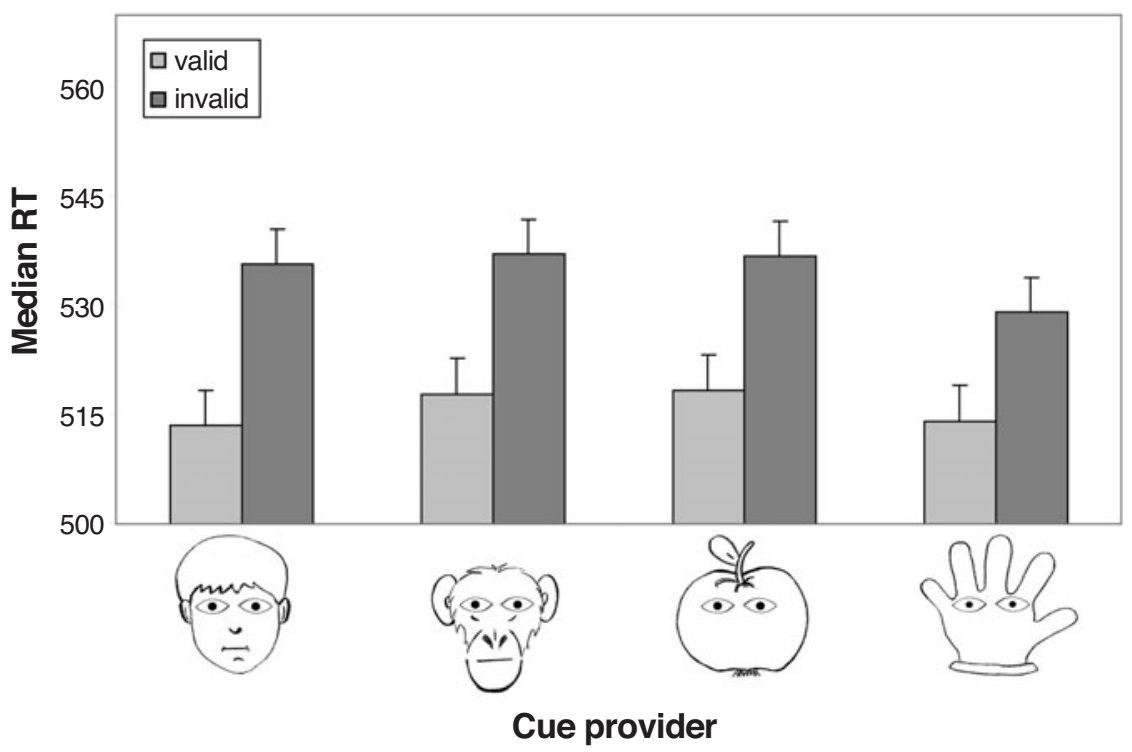

Figure 3. Median response time (in milliseconds) as a function of cue provider and trial type in Experiment 2. 
The experiment had a 2 (cue type: valid or invalid) $\times 2$ (cue status: eyes or arrows) repeated measures design.

Stimulus, Materials, and Procedure. The study was a replication of Experiments 1 and 2, but with a couple of modifications. The cue provider was always a glove, but the direction of visual attention was signaled by either eyes or arrows (i.e., arrows appeared in the same location as the eyes). On each trial, a fixation cross appeared in the center of the screen for $500 \mathrm{msec}$. This figure was then erased and replaced by a glove displaying direct gaze or a forward facing arrow $(500 \mathrm{msec})$, followed by the same glove displaying either the gaze or arrows pointing to the left or right (100 msec). A target letter ( $L$ or $T$ ) then appeared on either the left or right side of the screen and remained until participants made a response (i.e., cue/letter SOA $=100 \mathrm{msec}$ ). A reduced SOA (i.e., $100 \mathrm{msec}$ ) was used in this experiment to ensure that the observed effects were driven by automatic orienting (Müller \& Rabbitt, 1989). Participants completed 160 trials ( 80 valid trials, 40 eyes and 40 arrows, and 80 invalid trials, 40 eyes and 40 arrows), with order of presentation of the cue provider, gaze direction, and target location randomized across the experiment. On completion of the task, participants were debriefed, thanked, and dismissed.

\section{Results and Discussion}

Errors occurred on $5.3 \%$ of the trials and were excluded from the statistical analysis. Median response times were calculated for each of the experimental conditions and submitted to a 2 (cue type: valid or invalid) $\times$ 2 (cue status: eyes or arrows) repeated measures ANOVA. The analysis revealed a main effect of cue type $[F(1,9)=$ $5.09, p<.05]$, indicating that participants returned faster responses on cued than on noncued trials. In addition, a main effect of cue status was observed $[F(1,9=5.75$, $p<.04]$, such that responses were faster on trials depicting eye rather than arrow cues. The cue type $\times$ cue status interaction $[F(1,9)=1.47$, n.s. $]$ was not significant. These results reveal that the nature of the directional cue did not moderate attentional orienting (Ristic et al., 2002), since both eyes (i.e., a biologically relevant cue) and arrows (i.e., a biologically irrelevant cue) triggered exogenous shifts in visual attention (see Figure 4). Nevertheless, responding (on both valid and invalid trials) was facilitated when eyes rather than arrows were presented to participants, suggesting that gaze cues may enhance the overall efficiency of attentional processing.

\section{GENERAL DISCUSSION}

The present findings replicated previous research by demonstrating reflexive attentional orienting in response to gaze cues (Driver et al., 1999; Friesen \& Kingstone, 1998, 2003; Hietanen, 1999; Hood et al., 1998; Kingstone et al., 2000; Langton \& Bruce, 1999). In addition, however, they revealed that gaze-related shifts in visual attention were not moderated by the identity of the cue provider. Our analysis therefore argues against the viewpoint that the enhanced biological relevance of conspecifics may modulate the functioning of the human attentional system (Morton \& Johnson, 1991). Although perceivers routinely follow gaze cues provided by other people, in no sense is this effect dependent on the presence of a human cue provider. As demonstrated herein, comparable orienting effects can be elicited by gaze cues delivered by a chimpanzee, owl, or tiger (Experiment 1). Moreover, it would appear that the mere possession of eyelike stimuli is sufficient to trigger attentional orienting (Baron-Cohen, 1995; von Grünau \& Anston, 1995), as comparable effects were observed when gaze cues were provided by an apple or a glove (Experiment 2).

It is not only gaze cues, however, that trigger exogenous shifts in visual attention, for arrows also elicited visuospatial orienting (Experiment 3). By replicating

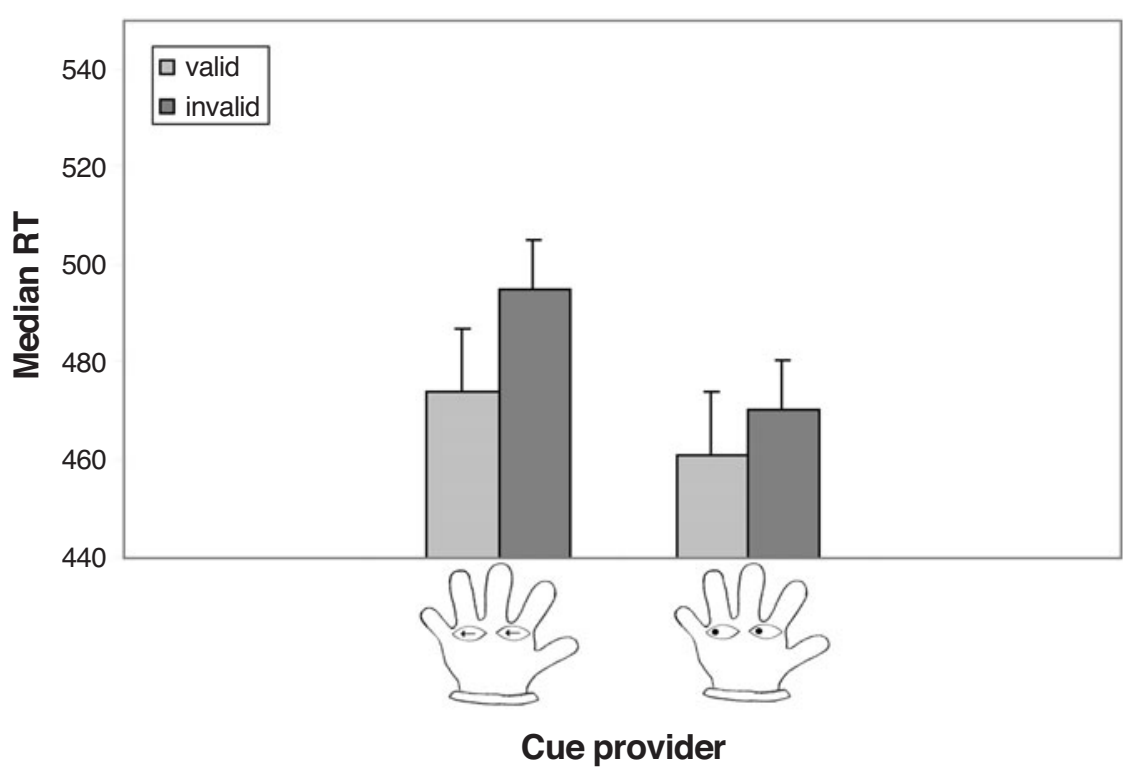

Figure 4. Median response time (in milliseconds) as a function of cue status and trial type in Experiment 3. 
previous research of this kind (Ristic et al., 2002; Tipples, 2002), this finding challenges the contention that gaze cues play a qualitatively distinct role in the human attentional system (Friesen \& Kingstone, 1998). Notwithstanding this observation, however, reflexive visual orienting may nevertheless represent an attentional process that is most efficient for biologically relevant stimulus cues, such as eyes (Baron-Cohen, 1995; Emery, 2000). In a recent investigation, Ristic et al. (2002) considered whether reflexive orienting is unique to biologically relevant stimuli (e.g., eyes), or can be triggered by other directional cues (e.g., arrows). They reported two noteworthy effects: First, reflexive visual orienting was elicited by both types of cue. Second, nonpredictive arrows produced reflexive orienting in both hemispheres of a splitbrain patient. The latter finding is important, as previous work by Kingstone et al. (2000) demonstrated that in split-brain patients, nonpredictive gaze cues produce reflexive orienting only in the hemisphere that is specialized for face processing. Thus, although both types of cue (i.e., biological and nonbiological) give rise to identical behavioral effects, they may not be supported by the same neural operations. Further work is needed to clarify this issue. At the behavioral level, the present findings further underscore the importance of biological cues in the functioning of the attentional system. Although both eyes and arrows prompted the emergence of visuospatial orienting, gaze cues served to enhance the overall efficiency of attentional processing. In this way, gaze cues may exert quantitative rather than qualitative effects on human attentional operations.

\section{REFERENCES}

Allison, T., Puce, A., \& McCarthy, G. (2000). Social perception from visual cues: Role of the STS region. Trends in Cognitive Sciences, 4, 267-278.

BARON-COHEN, S. (1995). Mindblindness: An essay on autism and theory of mind. Cambridge, MA: MIT Press.

Call, J., Hare, B., \& Tomasello, M. (1998). Chimpanzee gaze following in an object choice task. Animal Cognition, 1, 89-90.

Deaner, R. O., \& Platt, M. L. (2003). Reflexive social attention in monkeys and humans. Current Biology, 13, 1609-1613.

Driver, J., Davis, G., Ricciardelli, P., Kidd, P., Maxwell, E., \& BARON-COHEN, S. (1999). Gaze perception triggers visuospatial orienting by adults in a reflexive manner. Visual Cognition, 6, 509-540.

EMERY, N. J. (2000). The eyes have it: The neuroethology, function and evolution of social gaze. Neuroscience \& Biobehavioral Reviews, 24, $\underline{581-604 .}$

FriesEn, C. K., \& Kingstone, A. (1998). The eyes have it! Reflexive orienting is triggered by nonpredictive gaze. Psychonomic Bulletin \& Review, 5, 490-495.

Friesen, C. K., \& Kingstone, A. (2003). Abrupt onsets and gaze direction cues trigger independent reflexive attentional effects. Cognition, 87, 1-10.

GOREN, C. C., SARTY, M., \& Wu, P. Y. (1975). Visual following and pattern discrimination of face-like stimuli by newborn infants. Pediatrics, 56, 544-549.

HaXbY, J. V., HofFMAN, E. A., \& GobbInI, M. I. (2000). The distributed human neural system for face perception. Trends in Cognitive Sciences, 4, 223-233.

HIETANEN, J. K. (1999). Does your gaze direction and head orientation shift my visual attention? NeuroReport, 10, 3443-3447.
Hoffman, E. A., \& HaXby, J. V. (2000). Distinct representations of eye gaze and identity in the distributed human neural system for face processing. Nature Neuroscience, 3, 80-84.

Hood, B. M., Willen, J. D., \& DR̆IVER, J. (1998). Adults' eyes trigger shifts of visual attention in human infants. Psychological Science, $\mathbf{9}_{2}$ 131-134.

Johnson, M. H., Dziurawiec, S., Ellis, H., \& Morton, J. (1991). Newborns' preferential tracking of face-like stimuli and its subsequent decline. Cognition, 40, 1-19.

Kingstone, A., Friesen, C. K., \& Gazzaniga, M. S. (2000). Reflexive joint attention depends on lateralized cortical connections. Psychological Science, 11, 159-166.

Kingstone, A., SmileK, D., Ristic, J., Friesen, C. K., \& Eastwood, J. D. (2003). Attention, researchers! It is time to take a look at the real world. Current Directions in Psychological Science, 12, 176-180. KLEINKE, C. L. (1986). Gaze and eye contact: A research review. Psychological Bulletin, 100,78-100.

LANGTON, S. R. H., \& BRUCE, V. (1999). Reflexive social orienting. Visual Cognition, 6, 541-567.

Langton, S. R. H., WatT, R. J., \& Bruce, V. (2000). Do the eyes have it? Cues to the direction of social attention. Trends in Cognitive Sciences, 4, 50-59.

Macrae, C. N., Hood, B. M., Milne, A. B., Rowe, A. C., \& Mason, M. F. (2002). Are you looking at me? Eye gaze and person perception. Psychological Science, 13, 460-464.

Miklosi, A., Polgardi, R., Topal, J., \& Csanyi, V. (1998). Use of experimenter-given cues in dogs. Animal Cognition, 1, 113-121.

Morton, J., \& Johnson, M. H. (1991). CONSPEC and CONLERN: A two-process theory of infant face recognition. Psychological Review, 98, 164-181.

MǗLLR, H. J., \& RABBitT, P. M. A. (1989). Reflexive and voluntary orienting of visual attention: Time course of activation and resistance to interruption. Journal of Experimental Psychology: Human Perception \& Performance, 15,315-330.

Perrett, D. I., Hietanen, J. K., Oram, M. W., \& Benson, P. J. (1992). Organization and functions of cells responsive to faces in the temporal cortex. Philosophical Transactions of the Royal Society of London: Series $B, \mathbf{3 3 5}, 23-30$.

Perrett, D. I., Smith, P. A., Potter, D. D., Mistlin, A. J., Head, A. S., Milner, A. D., \& Jeeves, M. A. (1985). Visual cells in the temporal cortex sensitive to face view and gaze direction. Proceedings of the Royal Society of London: Series B, 223, 293-317.

PoSNER, M. I. (1980). Orienting of attention. Quarterly Journal of Experimental Psychology, 32, 3-25.

Povinelli, D. J., \& EDDY, T. J. (1996). Chimpanzees: Joint visual attention. Psychological Science, 7, 129-135.

Povinelli, D. J., \& EDDY, T. J. (1997). Specificity of gaze-following in young chimpanzees. British Journal of Developmental Psychology, 15, 213-222.

Puce, A., Allison, T., Bentin, S., Gore, J. C., \& McCarthy, G. (1998). Temporal cortex activation in humans viewing eye and mouth movements. Journal of Neuroscience, 18, 2188-2199.

Ristic, J., Friesen, C. K., \& Kingstone, A. (2002). Are eyes special? It depends on how you look at it. Psychonomic Bulletin \& Review, 9 , 507-513.

SCHULlER, A.-M., \& Rossion, B. (2001). Spatial attention triggered by eye gaze increases and speeds up early visual acuity. NeuroReport, 12, 2381-2386.

TipplEs, J. (2002). Eye gaze is not unique: Automatic orienting in response to uninformative arrows. Psychonomic Bulletin \& Review, 9, 314-318.

Tomasello, M., Call, J., \& HaRe, B. (1998). Five primate species follow the visual gaze of conspecifics. Animal Behaviour, 55, 1063-1069.

VON GRÜnaU, M., \& Anston, C. (1995). The detection of gaze direction: A stare-in-the-crowd effect. Perception, 24, 1297-1313.

Wicker, B., Michel, F., HenAfF, M. A., \& DeCETY, J. (1998). Brain regions involved in the perception of gaze: A PET study. Neurolmage, 8, 221-227.

(Manuscript received May 21, 2003; revision accepted for publication November 4, 2003.) 